\title{
Spatial representation by young infants: Categorization of spatial relations or sensitivity to a crossing primitive?
}

\author{
PAUL C. QUINN \\ University of Delaware, Newark, Delaware
}

\begin{abstract}
The spatial representation abilities of 3- to 4-month-old infants were examined in four experiments. Experiments 1 and 2 showed that infants familiarized with a diamond appearing in distinct locations to the left or right of a vertical bar subsequently preferred a stimulus depicting the diamond on the opposite side of the bar over a stimulus depicting the diamond in a novel location on the same side of the bar. Experiment 3 was a replication of Experiment 1, except that the bar was oriented at $45^{\circ}$. In this instance, infants divided their attention between the stimulus depicting the diamond on the opposite side of the bar and the stimulus depicting the diamond in a novel location on the same side of the bar. Experiment 4 demonstrated that the results of Experiment 3 were not a consequence of a failure to process the diagonal bar. When considered with previous reports that infants can represent the categories of above and below (Quinn, 1994), the present results suggest that (1) infants can also represent the categories of left and right, and (2) performance cannot be interpreted as a response to an arbitrary crossing of one object relative to another. Although recent discussions of the relation between language and cognition have pointed to the ways in which spatial language influences spatial cognition (Bowerman \& Levinson, 2001), the present findings are consistent with an influence in the opposite direction: Spatial cognition may in some instances shape spatial language.
\end{abstract}

Emerging evidence suggests that infants as young as 3 to 4 months of age possess core abilities to categorize two fundamental types of visual elements: objects and their spatial relations, or what and where information (Quinn, 2003). In the domain of objects, infants have been shown to categorize photographic exemplars of animals and furniture at both global and basic levels (Quinn, 2002b). In the domain of spatial relations, infants have been found to categorize above, below, and between (Quinn, in press). Early category representations in both domains are associated with adaptive processing that comes in the form of organized storage of information in memory, efficient retrieval of that information, and the capability of responding equivalently to an indefinitely large number of novel instances from multiple categories. They are also consistent with the idea that at least the initial beginnings of category formation may be unassisted by language (cf. Xu \& Carey, 1996), essentialist beliefs (cf. Gelman, 2003), or formal instruction.

This article will focus on the development of category representations in the domain of spatial relations. Comprehending the development of spatial representation has been a central task for the study of cognitive development since

This research was supported by NSF Grant BCS-0096300 and NIH Grant HD-42451. The author thanks Lila Gleitman, James E. Hoffman, Helene Intraub, Laura Wagner, and three anonymous reviewers for helpful discussion of the issues associated with this research. Address correspondence and reprint requests to P. C. Quinn, Department of Psychology, University of Delaware, Newark, DE 19716-2577 (e-mail: pquinn@udel.edu). the work of Piaget and Inhelder (1948/1967). Interest in understanding the emergence of spatial cognition has in the past led researchers to inquire whether there is a developmental transition from egocentric to allocentric coding of spatial location (Acredolo, 1978; Bremner \& Bryant, 1977; Rieser, 1979). More recently, Newcombe and Huttenlocher (2000) have proposed that the egocentric-to-allocentric shift may reflect quantitative changes in cue validities of various kinds of spatial coding rather than a qualitative change in the nature of spatial coding.

Another inquiry into the development of spatial coding has been based on investigations of whether or not infants are able to form and use concepts of spatial relations (Casasola \& Cohen, 2002; Casasola, Cohen, \& Chiarello, 2003; Hespos \& Baillargeon, 2001; McDonough, Choi, \& Mandler, 2003; Quinn, 1994; Quinn, Adams, Kennedy, Shettler, \& Wasnik, 2003; Quinn, Norris, Pasko, Schmader, \& Mash, 1999; Spelke \& Hespos, 2002). The findings are consistent with the idea that infants in the age range from 3 to 14 months are capable of representing smallscale spatial relations (e.g., above, below, and between), and physical concepts of spatial relations such as containment and support. Such representations should allow infants to experience objects in organized spatial arrangements, rather than as disconnected entities residing in unrelated locations.

One category distinction potentially available to young infants in the spatial domain that has been relatively understudied is that between left and right. An exception is the report of Behl-Chadha and Eimas (1995). These 
investigators familiarized 3- and 4-month-olds with stimulus displays depicting horse-zebra pairs presented in a constant left-right relation. Infants were then tested with a horse-zebra pair that maintained the familiar left-right relation paired with a horse-zebra pair presented in a novel right-left relation. The novel spatial relation was reliably preferred, a result consistent with the idea that the infants formed category representations for the left versus right relations of two objects. However, the infants could have processed the familiar stimuli not as two objects in a particular left-right relation, but rather as one holistic pattern. If this was the case, then performance on the test trials may have reflected pattern discrimination; that is, the infants may have discriminated the familiar pattern from one in which the two sides (striped for zebra and nonstriped for horse) were switched (i.e., the equivalent of a mirror image).

In the present research, the question asked was whether infants would form category representations for left versus right using a procedure that is more difficult to interpret in terms of simple pattern discrimination. The procedure was one that Quinn and colleagues have employed previously to investigate whether infants can form the category representations above versus below (Mash, Quinn, Dobson, \& Narter, 1998; Quinn, 1994; Quinn, Cummins, Kase, Martin, \& Weissman, 1996; Quinn, Polly, Furer, Dobson, \& Narter, 2002). In this procedure, 3- and 4-month-olds become familiarized with an object (i.e., a dot or diamond) located in different positions above or below a horizontal bar. In a subsequent paired preference test, the infants preferred a novel exemplar from the novel spatial category (i.e., an object located on the opposite side of the bar) to a novel exemplar from the familiar spatial category (i.e., an object located in a novel position on the same side of the bar). The findings could not be explained by simple generalization based on distance information, inasmuch as the object on the opposite side of the bar was positioned at the same distance from the familiar object locations as was the object in the novel location on the same side of the bar. An additional control experiment revealed that the infants could reliably discriminate the location changes that were depicted during familiarization. Also, the infants did not display a novel category preference in a further control experiment in which the object was presented in the different locations but without the bar. Considered together, the findings provide evidence that infants as young as 3 months of age can form category representations for the above and below arrangements of the object and horizontal bar.

The present study was designed to extend the findings demonstrating representation of above versus below, by examining whether infants could form category representations for the left and right arrangements of an object and a vertical bar in Experiments 1 and 2. In addition, in Experiments 3 and 4, an alternative interpretation for infant performance was considered, one in which infants were presumed to simply be responding to an arbitrary crossing of one object (e.g., a diamond) relative to another (i.e., the bar).

\section{EXPERIMENT 1}

In Experiment 1, the same procedure that was used to demonstrate that young infants could represent the above and below relations of an object and a horizontal bar was extended to investigate whether 3- to 4-month-olds could also form category representations for the left versus right relations of an object and a vertical bar. The design of Experiment 1 is shown in Figure 1. To investigate possible representation of the category "diamond left of bar," one group of infants was familiarized with four exemplars, each depicting a single diamond in a different position to the left of a vertical bar. Panels A and B of Figure 1 display the locations of the diamond as it appeared in the familiar exemplars. Half of the infants were presented with the diamond appearing in each of the four corner locations of an imaginary rectangle located in the top half of the area to the left of the bar (shown in panel A of Figure 1). Immediately following familiarization, test trials were administered in which a novel exemplar from the familiar category (e.g., diamond in new position to the left of bar) was paired with a novel exemplar from a
Familiar Stimuli

A.

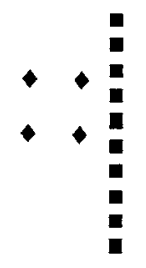

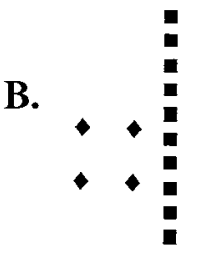

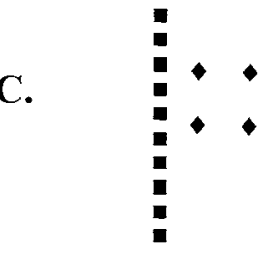

D.

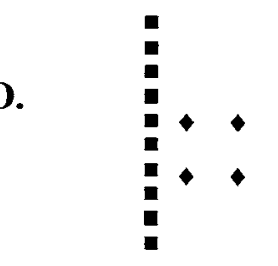

Test Stimuli
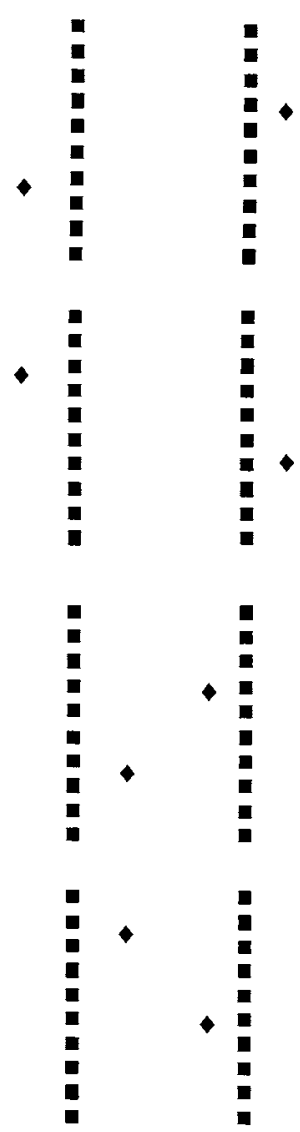

Figure 1. Familiarization stimuli (a composite of the four exemplars) and test stimuli used to determine whether infants form category representations for left (panels $A$ and $B$ ) or right (panels $C$ and D) in Experiment 1. 
novel category (e.g., diamond to the right of bar). It should be mentioned that the diamond in both test exemplars was moved the same distance from the average location of the diamond during familiarization (i.e., the center of the imaginary rectangle). In the case of the novel exemplar from the familiar category, the diamond was simply moved below the rectangle's center. For the novel exemplar from the novel category, the diamond was moved the same distance right (from the rectangle's center) and consequently to the right of the bar.

If infants can form a category representation for the spatial relation "diamond left of bar," they should display a preference for the novel exemplar from the novel category (i.e., the diamond to the right of the bar). However, if infants only process information about the diamond or the bar, or if they process the diamond and the bar independently of one another, a differential preference would not be expected. The other half of the infants that were tested for formation of the category representation "diamond left of bar" were familiarized with exemplars in which the diamond appeared in the four corner locations of an imaginary rectangle positioned in the bottom half of the area to the left of the bar (shown in panel B of Figure 1). Test trials followed in which a novel instance from the familiar category (diamond moved above the rectangle's center) was paired with a novel instance from a novel category (diamond moved the same distance to the right of the rectangle's center). Panels C and D of Figure 1 display comparable experimental sequences designed to investigate formation of the category representation "diamond right of bar" with a second group of infants.

\section{Method}

Participants. The participants were 24 healthy, full-term 3- to 4-month-old infants (mean age $=108.88$ days, $S D=6.09$ days). Fourteen were female and 10 were male. Three additional infants were tested; but 2 did not complete the procedure due to fussiness, and 1 was excluded from the data analysis due to position preference.

Apparatus. All infants in each experiment were tested in a visual preference apparatus, modeled on the one described by Fagan (1970). This apparatus is a large, three-sided gray viewing chamber on wheels. It has a hinged, gray display panel onto which were attached two compartments to hold posterboard stimuli. The compartments are situated in front of and to the left and to the right of the infant, thereby allowing simultaneous presentation of two stimuli, either two identical copies of the same stimulus (as was the case during the familiarization trials) or two different stimuli (as was the case during the preference test trials). The stimuli were illuminated by a fluorescent lamp that was shielded from the infant's view. The center-to-center distance between compartments was $30.5 \mathrm{~cm}$, and on all trials the display panel was situated approximately $30.5 \mathrm{~cm}$ in front of the infant. A $0.62-\mathrm{cm}$ peephole located midway between the two display compartments permitted an observer to record the infant's visual fixations. A second peephole, $0.90 \mathrm{~cm}$ in diameter and located directly below the first peephole, permitted a Pro Video CVC-120PH pinhole camera and a JVC video recorder to record the infants' gaze durations.

Stimuli. The stimuli were adapted from the stimuli used by Quinn et al. (1996; Quinn, Polly, et al., 2002) to study infants' representation of above versus below. Each stimulus was created by arranging a black diamond and vertical bar onto a $17.7 \times 17.7 \mathrm{~cm}$ white posterboard card (see Figure 1). The bar, $15.5 \mathrm{~cm}\left(26.9^{\circ}\right)$ long $\times$ $0.9 \mathrm{~cm}\left(1.7^{\circ}\right)$ wide, was positioned vertically along the center of the card and was composed of a column of individual square elements that were $0.9 \mathrm{~cm}\left(1.7^{\circ}\right)$ per side and spaced $0.6 \mathrm{~cm}\left(1.1^{\circ}\right)$ apart. The diamond, $0.9 \mathrm{~cm}\left(1.7^{\circ}\right)$ per side, appeared in different positions to the left or right of the bar.

Each infant was familiarized with the diamond appearing in each of the four corners of an imaginary rectangle located in one of the four quadrants of the stimulus card (left of bar, above horizontal midline; left, below; right, above; right, below). The familiar element locations are depicted on the left side of Figure 1. The centerto-center distance between the top and bottom elements of each rectangle was $5.0 \mathrm{~cm}\left(9.3^{\circ}\right)$, whereas the distance between the left- and right-side elements was $3.5 \mathrm{~cm}\left(6.5^{\circ}\right)$. The center-to-center distance between the diamonds closest to the bar and the bar itself was $2.0 \mathrm{~cm}\left(3.8^{\circ}\right)$. The location of the novel stimulus on the same side of the bar (i.e., novel exemplar from the familiar category) was $6.5 \mathrm{~cm}\left(12.0^{\circ}\right)$ below or above the average of the familiar diamond locations (e.g., the center of the imaginary rectangle); the location of the novel stimulus on the opposite side of the bar (i.e., the novel category exemplar) was $6.5 \mathrm{~cm}\left(12.0^{\circ}\right)$ to the right or left of the rectangle's center (shown on the far right side of Figure 1).

Procedure. All infants underwent the following general procedure. They were brought to the laboratory by a parent and seated in a reclining position on the parent's lap. There were two experimenters, both of whom were naive to the hypotheses under investigation. The first experimenter positioned the apparatus so that the midline of the infant's head was aligned with the midline of the display panel. When the display panel was open, the infant could see the experimenter from the midsection up, in addition to a portion of the room that was a light background color. The experimenter selected the appropriate stimuli as had previously been determined for the forthcoming trial and loaded them into the compartments of the display panel from a nearby table. The experimenter then elicited the infant's attention and closed the panel, thereby exposing the stimuli to the infant. The parent was unable to see the stimuli.

During each trial, the first experimenter observed the infant through the small peephole and recorded visual fixations to the left and right stimuli by means of two $605 \mathrm{XE}$ Accusplit electronic stop watches, one of which was held in each hand. The second experimenter timed the fixed duration of the trial and signaled the end of the trial. Between trials, the first experimenter opened the panel, changed the stimuli, obtained the infant's attention, centered his or her gaze, and closed the panel to begin the next trial. The first and second experimenters changed places for the test trials. The experimenter who presented stimuli and measured infant fixations during familiarization now measured trial duration and signaled the end of each test trial, whereas the second experimenter presented the test stimuli and measured infant fixations. The two experimenters changed roles across infants. The second experimenter was always naive with respect to the familiar category in Experiments 1 and 3 or the familiar stimulus in Experiments 2 and 4.

To handle possible side biases (i.e., position preferences) sometimes displayed by individual infants, over all the trials, both familiarization and test, the looking time to one side (i.e., left or right compartment) of the display stage could not exceed $95 \%$ of the total looking time in order for the infant to be included in the data analysis. In addition, on preference test trials, each infant was required to look at both of the stimuli. Interobserver reliability for 24 randomly selected infants participating in Experiments 1-4 was later determined by comparing the looking times measured by the experimenter using the center peephole and an additional naive observer recording the looking times off-line from videotape records, and averaged .92 .

Twelve infants were randomly assigned to each of two groups, defined by the familiar category, left or right. All infants in the left group were administered four 15 -sec familiarization trials. On each 
trial, these infants were presented with two identical copies of a pattern in which a diamond appeared to the left of the bar. For half of the infants in the left group, the diamond appeared, over the course of the four trials, in each of the four corners of an imaginary rectangle located in the top left quadrant of the stimulus card (panel A of Figure 1). The order of presentation of the four diamond positions was randomized for each infant over the course of the four familiarization trials. Immediately after the familiarization trials, two 10 -sec test trials were administered in which a novel stimulus from the familiar left category was paired with a novel stimulus from the novel right category. For the familiar category stimulus, the diamond appeared in a location below the center of the imaginary rectangle. For the novel category stimulus, the diamond was moved the same distance, but to a location to the right of the rectangle's center. It is important to note that the two test stimuli were in one sense equally novel, in that the diamond was moved an equivalent distance away from the average position of the diamond during familiarization. The left-right positions of the familiar and novel category stimuli were counterbalanced across infants on the first test trial and reversed on the second test trial. For the other half of the infants in the left group, the familiarization trials had the diamond appearing in the four corner locations of an imaginary rectangle positioned in the bottom left quadrant of the stimulus card (panel B of Figure 1). These infants were tested with a familiar category exemplar in which the diamond appeared above the rectangle's center and a novel category exemplar in which the diamond was located the same distance to the right of the rectangle's center. The right group testing conditions (shown in panels C and D of Figure 1) were symmetric to those of the left group.

\section{Results and Discussion}

Familiarization trials. Individual looking times were summed over the left and right copies of the stimulus presented on each familiarization trial and were then averaged across the first two trials and last two trials. The mean looking times are shown in Table 1. An analysis of variance (ANOVA) with factors of trial block (1-2 vs. 3-4) $\times$ familiarization condition (left vs. right), performed on the individual looking time scores revealed a reliable effect only of trial block $[F(1,22)=4.56, p<$ $.05]$. No other effects were significant $(p>.05)$, in each instance. Using the standard operational definition of habituation as a decline in responsiveness with repeated stimulation (see, e.g., Cohen \& Gelber, 1975), the reliable decrement in looking time across familiarization trials indicates that infants in the left and right familiarization groups had habituated to the stimuli.

Preference test trials. Each infant's looking time to the novel category stimulus was divided by the looking

Table 1

Mean Fixation Times (in Seconds) During the Familiarization Trials and Mean Novel Category Preference Scores (Percentages) During the Preference Test Trials in Experiment 1

Fixation Time

\begin{tabular}{|c|c|c|c|c|c|c|c|}
\hline \multirow{2}{*}{$\begin{array}{c}\text { Familiarization } \\
\text { Category }\end{array}$} & \multicolumn{2}{|c|}{ Trials $1-2$} & \multicolumn{2}{|c|}{ Trials 3-4 } & \multicolumn{3}{|c|}{ Novelty Preference } \\
\hline & $M$ & $S D$ & $M$ & $S D$ & $M$ & $S D$ & $t^{\mathrm{a}}$ \\
\hline & & 282 & & 2. & 0 & & \\
\hline & & 3. & 8. & 3.69 & & & $3.04 !$ \\
\hline Combined & 9.38 & 3.02 & 8.51 & 3.20 & 62.05 & 15.03 & $3.93 \S$ \\
\hline
\end{tabular}

Note $-t$ a for mean versus chance. $\pitchfork p<.025$, one-tailed. $\ddagger p<.01$, one-tailed. $\$ p<.0005$, one-tailed. time to both test stimuli and then converted to a percentage score. The mean novel category preference scores for the left and right familiarization conditions are shown in Table 1. As can be seen, $t$ tests comparing the preference scores with $50 \%$ revealed the performance of both groups of infants to be reliably above chance, and the two groups did not differ from each other in this respect $[t(22)=-0.01, p>.20$, two-tailed]. Overall, the findings indicate that by $3-4$ months of age, infants can form distinct category representations for the left versus right relations of a diamond and a vertical bar.

\section{EXPERIMENT 2}

Reliable novel category preference scores can only be inferred as evidence for categorization if it is known that the individual exemplars of the familiar category are discriminably different for the participants (Quinn, 2002a). If the stimuli are not discriminable, a categorization experiment reduces to a test of discrimination between the nondiscriminable members of the familiar category (which become effectively one stimulus) and the novel exemplar from the novel category. Experiment 2 was thus conducted to determine whether the individual instances from within the left and right categories were discriminably different.

The procedure used to measure discriminability involved presenting a randomly selected exemplar from within the left or right categories for a single 15-sec trial. A single 15-sec trial was used because it corresponded with the length of time that each exemplar was presented during familiarization in the test of categorization in Experiment 1 (Quinn, 1998). Immediately following familiarization, the familiar stimulus was paired with a novel exemplar from the same category, also randomly selected, for two 10-sec test trials. Panels A and B of Figure 2 display representative experimental sequences for discrimination within the left and right categories.

\section{Method}

Participants. The participants were 24 healthy, full-term 3- to 4 -month-old infants with a mean age of 110.46 days $(S D=8.47)$. There were 12 boys and 12 girls. Three additional infants were tested, but 2 did not complete the procedure due to fussiness and 1 was excluded from the data analysis because of position preference.

Stimuli. The stimuli were the familiar category stimuli used in Experiment 1.

Procedure. The within-category discrimination tests followed from the categorization tests of Experiment 1. In Experiment 1, each infant was familiarized with four exemplars, each depicting a diamond positioned in one of four corners of an imaginary rectangle located in one of the four quadrants on the stimulus card. The novel instance of the familiar category shown during test trials contained a diamond shifted below or above the diamond locations shown during familiarization. In Experiment 2, each infant was tested for discrimination between two of the five exemplars used to represent the familiar category in a given familiarization and preference test condition in Experiment 1. The pair of exemplars was randomly chosen for each infant, as was which member of the pair would serve as the familiar stimulus. Familiarization consisted of a single 15-sec familiarization trial, during which time the familiar 


\section{Familiar Stimuli}

A.

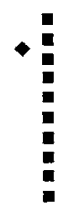

B.

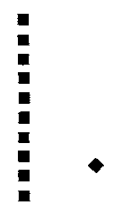

\section{Test Stimuli}
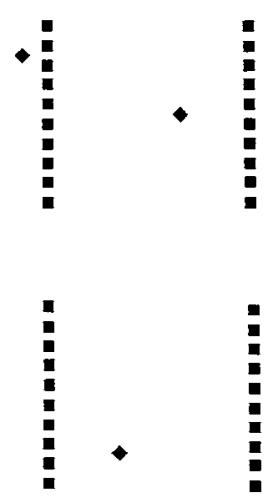

Figure 2. Representative experimental sequences used to test discrimination within the left (panel A) and right (panel B) categories in Experiment 2.

stimulus was shown in both compartments of the display stage. Immediately after familiarization, the familiar stimulus was paired with the novel stimulus for two 10 -sec test trials. The left-right positioning of the novel stimulus was counterbalanced across infants on the first test trial and reversed on the second test trial. Half of the participants were tested with the diamond locations to the left of the bar, and the other half were tested with diamond positions to the right of the bar.

\section{Results and Discussion}

Familiarization trial. Individual looking times were summed over the left and right copies of the stimulus on the familiarization trial and then averaged across infants. Mean looking times to the stimuli presented to the left of the bar and to the right of the bar are shown in Table 2. The difference in mean looking times was not significant $[t(22)=-0.16, p>.20$, two-tailed $]$.

Preference test trials. Each infant's looking time to the novel stimulus was divided by the looking time to both test stimuli and then converted to a percentage score. The mean preference scores for the novel stimulus for the left and right familiarization conditions are shown in Table 2. The means were well above $50 \%$, and $t$ tests comparing the mean scores to chance revealed both to be statistically reliable. In addition, a $t$ test showed that the two mean scores were not reliably different from each other $[t(22)=0.58, p>.20$, two-tailed]. Infants familiarized with a stimulus depicting the diamond to either

Table 2

Mean Fixation Times (in Seconds) During the Familiarization Trial and Mean Novelty Preference Scores (Percentages) During the Preference Test Trials in Experiment 2

\begin{tabular}{lcccrrr}
\hline \multirow{2}{*}{$\begin{array}{l}\text { Experimental } \\
\text { Group }\end{array}$} & \multicolumn{2}{c}{ Fixation Time } & & \multicolumn{3}{c}{ Novelty Preference } \\
\cline { 2 - 4 } \cline { 5 - 7 } & $M$ & $S D$ & & $M$ & \multicolumn{1}{c}{$S D$} & $t^{\text {a }}$ \\
\hline Left & 8.99 & 2.53 & & 59.72 & 18.50 & $1.82 *$ \\
Right & 9.16 & 2.67 & & 56.29 & 8.70 & $2.50 \dagger$ \\
Combined & 9.08 & 2.54 & 58.00 & 14.25 & $2.75 \ddagger$ \\
\hline
\end{tabular}

Note $-t$ a for mean versus chance. $* p<.05$, one-tailed. ${ }^{\dagger} p<.025$, one-tailed. $\ddagger p<.01$, one-tailed. the left or the right of the bar preferred a stimulus depicting the diamond in a novel position on the same side of the bar. These results indicate that 3- and 4-montholds were able to discriminate the exemplars from within the left and right categories, and that the novel category preference scores observed in Experiment 1 were unlikely to have arisen from within-category discrimination failure.

\section{EXPERIMENT 3}

Despite the reports that young infants can represent above versus below (Mash et al., 1998; Quinn, 1994; Quinn et al., 1996; Quinn, Polly, et al., 2002) and left versus right (Experiment 1 of the present study), there is one unresolved issue with respect to the interpretation of performance. Specifically, it is possible that infants are simply responding to the crossing of an object from one side of a bar to the other (Landau \& Jackendoff, 1993; Talmy, 1983), rather than representing the spatial relations above, below, left, and right. To investigate this possibility, Experiment 3 used the categorization experimental design of Experiment 1; however, the bar was oriented at $45^{\circ}$, rather than at $0^{\circ}$ (horizontal) or $90^{\circ}$ (vertical) as it was in the previous studies. The experimental sequences are depicted in Figure 3. If the infants are responding only to the crossing of a target object (e.g., diamond) with respect to a referent object (e.g., bar), then a preference for the novel spatial relation should be maintained. However, if the infants in the previous studies were representing spatial relations such as above, below, left, and right, we would not expect a preference for the novel spatial relation, given that the crossing in this case is more arbitrary, and does not map cleanly onto a fundamental spatial concept.

\section{Method}

Participants. The participants were 24 healthy, full-term 3- to 4-month-old infants with a mean age of 110.92 days $(S D=9.63)$. There were 10 boys and 14 girls. Two additional infants were tested but did not complete the procedure due to fussiness.

Stimuli. The stimuli were the same as those used in Experiment 1 , except that the bar was oriented at $45^{\circ}$ counterclockwise from vertical, as is shown in Figure 3.

Procedure. Experiment 3 employed the same experimental design as Experiment 1 with the exception that the familiar categories were below-left and above-right.

\section{Results and Discussion}

Familiarization trials. Mean looking times for the below-left and above-right familiarization conditions are shown in Table 3. An ANOVA with the factors of trial block $\times$ familiarization condition (below left vs. above right) performed on the individual scores revealed a significant effect only of trial block $[F(1,22)=5.43, p<$ $.03]$, indicating an overall decline in looking time from the first to the second half of familiarization. The reliable decrement in looking time across familiarization trials indicates that infants in both groups had habituated to the stimuli. 


\section{Familiar Stimuli}

\section{Test Stimuli}

A.
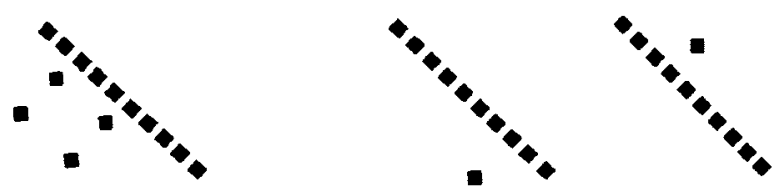

B.
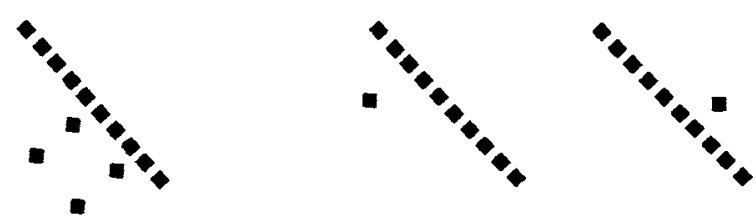

C.
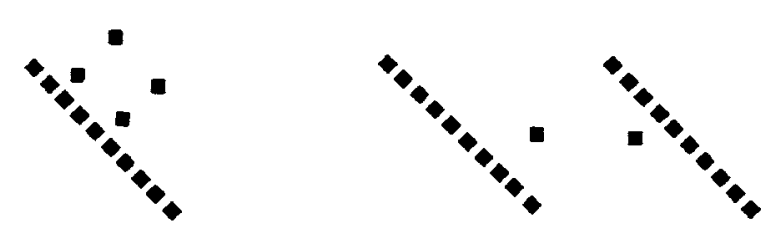

D.
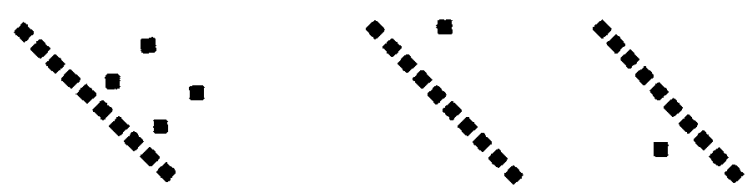

Figure 3. Familiarization stimuli (a composite of the four exemplars) and test stimuli used to determine whether infants form category representations for below left (panels $A$ and $B$ ) and above right (panels $C$ and D) in Experiment 3.

Preference test trials. Table 3 displays the mean novel category preference scores for the below-left and aboveright familiarization conditions. Neither score differed significantly from chance, and the scores did not differ significantly from each other $[t(22)=-0.73, p>.20$, two-tailed]. These results indicate that infants do not reliably respond to a change in spatial relation information when the bar is oriented at $45^{\circ}$, and the diamond crosses from one side to the other (e.g., below left to above right) in the transition from familiarization to the novel category preference test. The findings imply that the previous studies investigating the categorization of spatial relation information by infants were likely demonstrating that infants can represent above versus below (Mash et al., 1998; Quinn, 1994; Quinn et al., 1996) and left versus right (Experiment 1 of the present study), rather than simply responding to a crossing of one element (e.g., diamond) from one to the other side of another element (e.g., bar).

\section{EXPERIMENT 4}

Experiment 4 explored one possible reason why the infants in Experiment 3 did not respond preferentially to the novel category exemplars. In particular, it examined whether the infants were able to encode the diagonality of the bar. If infants could not encode the orientation of the bar, they clearly would not be able to respond to crossings of the object from one side of the bar (e.g., below left) to the other (e.g., above right). To investigate this possibility, infants were familiarized with the bar oriented at $45^{\circ}$ or $135^{\circ}$ and were then tested with a $45^{\circ}$ bar paired with a $135^{\circ}$ bar. This experimental design is depicted in panels A and B of Figure 4. If infants could

Table 3

Mean Fixation Times (in Seconds) During the Familiarization Trials and Mean Novel Category Preference Scores (Percentages) During the Preference Test Trials in Experiment 3

Fixation Time

\begin{tabular}{|c|c|c|c|c|c|c|c|}
\hline \multirow{2}{*}{$\begin{array}{c}\text { Familiarization } \\
\text { Category }\end{array}$} & \multicolumn{2}{|c|}{ Trials $1-2$} & \multicolumn{2}{|c|}{ Trials 3-4 } & \multicolumn{3}{|c|}{ Novelty Preference } \\
\hline & $M$ & $S D$ & $M$ & $S D$ & $M$ & $S D$ & $t^{\mathrm{a}}$ \\
\hline & & & & & & & \\
\hline & & & & 3. & & & \\
\hline Combined & 9.64 & 2.66 & 8.41 & 2.77 & 48.21 & 12.92 & -0.68 \\
\hline
\end{tabular}

Note $-t^{\mathrm{a}}$ for mean versus chance. 


\section{Familiar Stimuli}

A.

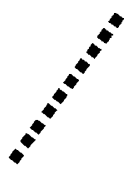

B.
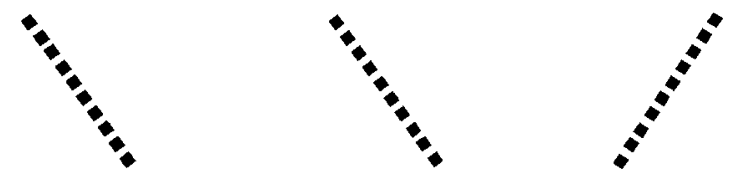

Figure 4. Familiarization and test stimuli used to measure discrimination of the two diagonals in Experiment 4.

not encode the diagonality of the bar, one would not expect a differential preference. However, if infants could encode the orientation of the bar, they should display a preference for the novel diagonal.

\section{Method}

Participants. The participants were 24 healthy, full-term 3- to 4-month-old infants with a mean age of 110.00 days $(S D=9.51)$. There were 10 boys and 14 girls. One additional infant was tested but did not complete the procedure due to fussiness.

Stimuli. Only two stimuli were needed for Experiment 4. One was the stimulus depicting the bar oriented at $45^{\circ}$ counterclockwise from vertical (the same orientation of the bar that was presented in Experiment 3), but without the diamond. The other stimulus was a bar oriented at $135^{\circ}$ counterclockwise from vertical. The stimuli are shown in Figure 4.

Procedure. Each infant was familiarized with the bar oriented at $45^{\circ}$ or $135^{\circ}$ for four $15-\mathrm{sec}$ familiarization trials. The infants were then presented with the $45^{\circ}$ bar paired with the $135^{\circ}$ bar on two 10 -sec test trials. The left-right positioning of the novel stimulus was counterbalanced across infants on the first test trial and reversed on the second test trial. Half of the participants were familiarized with the $45^{\circ}$ bar, and the other half with the $135^{\circ}$ bar.

\section{Results and Discussion}

Familiarization trials. Mean looking times for the $45^{\circ}$ and $135^{\circ}$ familiarization conditions are shown in Table 4. An ANOVA with the factors of trial block $\times$ familiar stimulus $\left(45^{\circ}\right.$ vs. $\left.135^{\circ}\right)$ performed on the individual scores revealed only a significant effect of trial block $[F(1,22)=8.82, p=.007]$, indicating an overall decline

Table 4

Mean Fixation Times (in Seconds) During the Familiarization Trials and Mean Novelty Preference Scores (Percentages) During the Preference Test Trials in Experiment 4

\begin{tabular}{|c|c|c|c|c|c|c|c|}
\hline \multirow{3}{*}{$\begin{array}{c}\text { Familiarization } \\
\text { Category }\end{array}$} & \multicolumn{4}{|c|}{ Fixation Time } & & & \\
\hline & \multicolumn{2}{|c|}{ Trials $1-2$} & \multicolumn{2}{|c|}{ Trials 3-4 } & \multicolumn{3}{|c|}{ Novelty Preference } \\
\hline & $M$ & $S D$ & $M$ & $S D$ & $M$ & $S D$ & $t^{\mathrm{a}}$ \\
\hline $45^{\circ}$ & 9.82 & 2.37 & 8.51 & 2.59 & 62.42 & 18.37 & $2.34 \dagger$ \\
\hline $135^{\circ}$ & 10.61 & 2.73 & 9.87 & 2.94 & 60.77 & 17.64 & $2.11 *$ \\
\hline Combined & 10.22 & 2.35 & 9.19 & 2.80 & 61.60 & 17.63 & $2.63 \ddagger$ \\
\hline
\end{tabular}

Note $-t$ a for mean versus chance. $\quad * p<.05$, one-tailed. ${ }^{\dagger} p<.025$, one-tailed. $\quad \ddagger p<.01$, one-tailed. in looking time from the first to the second half of familiarization. The reliable decrement in looking time across familiarization trials indicates that infants in both groups had habituated to the stimuli.

Preference test trials. Table 4 displays the mean novelty preference scores for the $45^{\circ}$ and $135^{\circ}$ familiarization conditions. As can be seen, $t$ tests comparing the preference scores with the chance preference of $50 \%$ revealed that infants in both groups reliably preferred the novel stimulus and that the two preference scores were not significantly different from each other $[t(22)=0.22$, $p>.20$, two-tailed]. These results suggest that infants were able to encode the diagonality of the bar when presented with the categorization task used in Experiment 3. The null results obtained in Experiment 3 were thus not likely to have occurred because of failure to encode the orientation of the bar.

\section{GENERAL DISCUSSION}

The four experiments reported here provide an important extension and clarification of previous research on young infant categorization of spatial relation information. In particular, an earlier study reporting that infants could represent left and right as distinct categories left open the possibility that the infants were simply engaging in pattern discrimination (Behl-Chadha \& Eimas, 1995), and prior reports that infants could represent above and below as individuated categories were also consistent with the idea that the infants were merely responding to the crossing of a target object relative to a referent bar (Mash et al., 1998; Quinn, 1994; Quinn et al., 1996; Quinn, Polly, et al., 2002).

\section{Experiments 1 and 2}

In Experiment 1, 3- to 4-month-old infants who were familiarized with a series of exemplars, each depicting a diamond in distinct locations to the left of a vertical reference bar, displayed a novel category preference for an exemplar displaying the diamond to the right of the bar. Likewise, infants familiarized with the diamond appearing in multiple positions to the right of the bar showed a preference for a stimulus in which the diamond was located to the left of the bar. Experiment 2 showed that the preferences of Experiment 1 were not a result of failure to discriminate the exemplars chosen from within the left and right categories. Taken together, the findings suggest that the infants were able to form category representations of the spatial relations left and right, and to respond to novel object arrangements on the basis of these representations.

An important feature of Experiments 1 and 2 is that they overcome a potential objection to the Behl-Chadha and Eimas (1995) report - namely, that infants might be representing the entire stimulus display as an abstract configuration and then responding to the spatial relation categorization task as if it were a mirror-image pattern discrimination task. At least two aspects of the stimulus displays used in the present study, along with prior find- 
ings suggesting that infants are sensitive to Gestalt grouping principles (e.g., Quinn, Bhatt, Brush, Grimes, \& Sharpnack, 2002; Quinn, Brown, \& Streppa, 1997; Quinn, Burke, \& Rush, 1993; Quinn \& Schyns, 2003), work against this possibility here. First, the squares making up the vertical bar were close to the other squares, but farther away from the diamond. Thus, via proximity, the squares should have grouped together as the bar, and the diamond should have remained distinct. Second, although the squares could be said to have had the same shape as the diamond, their orientation was different. Thus, via orientation similarity (Beck, 1966; Olson \& Attneave, 1970), the squares should again have grouped together to form the bar, and the diamond should have remained distinct. Thus, by virtue of proximity and orientation similarity, the squares would have been likely to group with the other squares and unlikely to group with the diamond. These factors suggest that the infants perceived the diamond and the bar depicted in Experiments 1 and 2 not as a single configuration, but rather as spatially distinct entities.

\section{Experiments 3 and 4}

In Experiment 3, 3- and 4-month-old infants who were familiarized with a series of exemplars, to the left of and below a diagonal bar, did not display a novel category preference for an exemplar in which the diamond appeared to the right of and above the bar. Similarly, infants familiarized with the diamond appearing in multiple locations to the right of and above the diagonal bar did not display a subsequent preference for a stimulus in which the diamond was positioned to the left of and below the bar. Experiment 4 showed that the null preference results were not the result of a failure to process the diagonality of the bar; infants familiarized with the bar oriented at $45^{\circ}$ or $135^{\circ}$ counterclockwise from vertical and then preference tested with both orientations showed a novelty preference for the novel orientation.

\section{Categorization of Spatial Relations or Sensitivity to a Crossing Primitive?}

The combined results of Experiments 3 and 4 are relevant to the interpretation of Experiments 1 and 2 as well as previous results reporting that infants can represent an object as being above or below a horizontal bar (Mash et al., 1998; Quinn, 1994; Quinn et al., 1996; Quinn, Polly, et al., 2002). Specifically, in Experiments 1 and 2 of the present study, and in all previous investigations of infants' representation of above versus below, the change from one spatial relation to another (i.e., from left to right or right to left and from above to below or below to above) was confounded with an object crossing from one side to the other side of a reference bar. This aspect of the experimental design thus left open the question of whether the infants might simply be responding to any crossing of a target object from one side to the other side of a reference bar. However, for that interpretation to hold true, one would have expected infants in Experi- ment 3 to respond to the change in the location of the diamond from one side to the other side of the bar during the transition from familiarization to preference test. The fact that the infants did not respond with a reliable novel category preference is consistent with the idea that the infants in Experiment 1 and in the earlier studies were representing the spatial relations left versus right and above versus below rather than responding to a primitive crossing concept.

\section{Development of Spatial Coding}

The cognitive science literature is rife with dual-system or dual-mode accounts of spatial coding in adults: Proffitt's perception-action versus cognitive system (Creem \& Proffitt, 1998), Sholl's self-to-object and object-to-object systems (Easton \& Sholl, 1995; Sholl \& Nolin, 1997), and Huttenlocher's fine-grained versus categorical coding (Huttenlocher, Hedges, \& Duncan, 1991). One may ask where the present results fit into this contemporary theoretical scene. The findings reported here are most consistent with cognitive and neural models of spatial coding that posit systems for processing (1) categorical spatial relations between objects on the one hand (e.g., above, below, left, right), and (2) finer grained spatial relations between objects on the other hand (e.g., object in one location to the left of bar vs. object in another location to the left of bar; Huttenlocher et al., 1991; Kosslyn et al., 1989). Evidence was obtained for the processing of categorical spatial information in Experiment 1 and diamond position information in Experiment 2. The data thus suggest that the fundamental components of the Huttenlocher et al. "categories and particulars" model of spatial coding proposed to account for spatial coding in adults are also present as starting points in young infants.

\section{Role of the Bar as a Referent}

A lingering issue centers on the role of the bar as providing an external referent in the present studies. Although the bar was present in Experiments 1-4, its role in producing the observed pattern of performance is considered crucial. This is because in earlier investigations in the same series, those that focused on above versus below (e.g., Quinn, 1994), the preference for the novel spatial relation during the test trials was not observed in a control experiment in which the target object (e.g., dot or diamond) was presented in the various locations but without the horizontal reference bar. Extending this result to the present studies, one may presume that the infants were encoding the diamond locations in relation to the externally available vertical reference bar rather than, for example, an internally generated vertical line that would bisect the stimulus display into left versus right regions (cf. Huttenlocher, Newcombe, \& Sandberg, 1994).

What remains to be resolved is why the vertical bar, but not the diagonal bar, was used as a referent. The processing of the bar itself could be at issue, or the processing of direction away from the bar, or both. Although Experiment 4 indicates that processing of the diagonal ori- 
entation of the bar was not a problem for the infants, it could also be that infants are less likely to use the bar as a referent. In connection with this idea, it should be mentioned that Huttenlocher et al. (1991) showed that adults remember the location of points in a two-dimensional space in terms of categories marked by the horizontal and vertical axes of the space, rather than the diagonal axes of the space. Moreover, Rosch (1975) used a stimulus placement task to show that horizontal and vertical, but not diagonal, serve as cognitive referents for organizing spatial orientation information. Specifically, $10^{\circ}$ deviations away from horizontal and vertical (but not diagonal) were placed closer to horizontal and vertical than horizontal and vertical were placed to the $10^{\circ}$ deviations. The combined findings from the present study indicating that young infants can represent left versus right categories relative to a vertical axis (but do not represent the above left vs. below right categories relative to a diagonal axis) as well as the previous reports that infants can represent above and below categories relative to a horizontal axis suggest that the use of horizontal and vertical axes (but not the diagonal axes) for the categorical coding of spatial information may be present from the beginnings of cognitive development. As such, the horizontal and vertical axes may serve as cognitive referents that provide the spatial domain with structure even in early infancy.

With respect to the idea that processing of direction away from the diagonal relative to the vertical could be at issue, the results reported here with young infants may have a correspondence with those observed in young children (Clark, 1972; Hoffman, Landau, \& Pagani, 2003). In particular, young children, in tasks ranging from block placement to lexical acquisition of spatial terms, are able to represent orientation or axis information (horizontal, vertical, and even diagonal in some instances), but they can display fragility in representing spatial relations surrounding such axes (see also McCloskey \& Rapp, 2000, for a related finding in adults). The present results are consistent with the idea that during the first few months of life such fragility is particularly manifest in the coding of direction away from the diagonal orientation or axis. In general, the findings suggest that young infants do not equivalently encode direction away from the major axes.

\section{Implications for the Relation Between Language and Space}

Accounting for the acquisition of the semantics of spatial relation concepts has turned out to be an extremely complex undertaking, with debate centering on the relative contributions of nonlinguistic perception and cognition versus language-specific semantic structure (Bowerman, 1996; Crawford, Regier, \& Huttenlocher, 2000; Hayward \& Tarr, 1995; Regier \& Carlson, 2001), as well as whether language plays a selectionist (Mandler, 1996), interactionist (Bowerman \& Choi, 2001) or combinatorial (HermerVazquez, Spelke, \& Katsnelson, 1999) role in the overall process. Much of the recent thinking in this area can be described as neo-Whorfian in perspective, with language acquisition helping to structure the concepts being formed (Bowerman \& Levinson, 2001). The present data are consistent with the idea that although infants are powerful learners of spatial relation concepts (Elman et al., 1996), they do not learn all relations equally well. Crossing over the diagonal is more difficult for infants to learn than above versus below and left versus right. The nonlinguistic differences may have a correspondence with later linguistic differences, in that children learning English eventually come to comprehend spatial terms such as above, below, left, and right, whereas English does not have any simple spatial terms to express direction away from the diagonal. More generally, just as some aspects of language acquisition may shape concept formation, some aspects of concept formation may shape language acquisition. As Landau (2003) has suggested, "The basic spatial terms - those that are monomorphemic - are few in number, and they appear to capture a distinctive set of spatial relationships . . these spatial "meanings" might have originated in the way the brain encodes location nonlinguistically" (p. 19). The findings reported here provide support for Landau's observation and indicate that the ability to represent some spatial relations (i.e., above vs. below a horizontal axis or left vs. right of a vertical axis) as more fundamental than others (above right vs. below left of a diagonal axis) may be derived from the initial settings of our perceptual-cognitive system.

\section{REFERENCES}

ACredolo, L. P. (1978). Development of spatial orientation in infancy. Developmental Psychology, 14, 224-234.

BECK, J. (1966). Effect of orientation and of shape similarity on perceptual grouping. Perception \& Psychophysics, 1, 300-302.

Behl-Chadha, G., \& Eimas, P. D. (1995). Infant categorization of left-right spatial relations. British Journal of Developmental Psychology, 13, 69-79.

BOWERMAN, M. (1996). Learning how to structure space for language: A cross-linguistic perspective. In P. Bloom, M. A. Peterson, L. Nadel, \& M. F. Garrett (Eds.), Language and space (pp. 385-436). Cambridge, MA: MIT Press.

Bowerman, M., \& CHOI, S. (2001). Shaping meanings for language: Universal and language-specific in the acquisition of spatial semantic categories. In M. Bowerman \& S. C. Levinson (Eds.), Language acquisition and conceptual development (pp. 475-511). Cambridge: Cambridge University Press.

Bowerman, M., \& LEvinson, S. C. (EDS.) (2001). Language acquisition and conceptual development. Cambridge: Cambridge University Press.

Bremner, J. G., \& Bryant, P. E. (1977). Place versus response as the basis of spatial errors made by young infants. Journal of Experimental Child Psychology, 23, 162-171.

Casasola, M., \& CoHen, L. B. (2002). Infant categorization of containment, support, and tight fit spatial relations. Developmental Science, 5, 247-264.

Casasola, M., Cohen, L. B., \& Chiarello, E. (2003). Six-month-old infants' categorization of containment spatial relations. Child Development, 74, 679-693.

CLARK, E. (1972). On the child's acquisition of antonyms in two semantic fields. Journal of Verbal Learning \& Verbal Behavior, 11, 750-758.

Cohen, L. B., \& Gelber, E. R. (1975). Infant visual memory. In L. B. Cohen \& P. Salapatek (Eds.), Infant perception: From sensation to cognition (Vol. 1, pp. 347-403). New York: Academic Press.

Crawford, L. E., Regier, T., \& Huttenlocher, J. (2000). Linguistic and non-linguistic spatial categorization. Cognition, 75, 209-235.

Creem, S. H., \& ProffitT, D. R. (1998). Two memories for geographical slant: Separation and interdependence of action and awareness. Psychonomic Bulletin \& Review, 5, 22-36. 
EAston, R. D., \& Sholl, M. J. (1995). Object-array structure, frames of reference, and retrieval of spatial knowledge. Journal of Experimental Psychology: Learning, Memory, \& Cognition, 21, 483-500.

Elman, J. L., Bates, E. A., Johnson, M. H., Karmiloff-Smith, A., Parisi, D., \& Plunkett, K. (1996). Rethinking innateness: A connectionist perspective on development. Cambridge, MA: MIT Press.

FAGAN, J. F. (1970). Memory in the infant. Journal of Experimental Child Psychology, 9, 217-226.

Gelman, S. A. (2003). The essential child: Origins of essentialism in everyday thought. New York: Oxford University Press.

HAYWARD, W. G., \& TARR, M. J. (1995). Spatial language and spatial representation. Cognition, 55, 39-84.

Hermer-VAzQuez, L., SPel Ke, E. S., \& Katsnelson, A. S. (1999). Sources of flexibility in human cognition: Dual-task studies of space and language. Cognitive Psychology, 39, 3-36.

Hespos, S. J., \& BaillargeOn, R. (2001). Infants' knowledge about occlusion and containment events: A surprising discrepancy. Psychological Science, 12, 141-147.

HoffMAN, J. E., LANDAU, B., \& PAGANI, B. (2003). Spatial breakdown in spatial construction: Evidence from eye fixations in children with Williams syndrome. Cognitive Psychology, 46, 260-301.

Huttenlocher, J., Hedges, L. V., \& DuncaN, S. (1991). Categories and particulars: Prototype effects in estimating spatial location. $\underline{P s y-}$ chological Review, 98, 352-376.

Huttenlocher, J., Newcombe, N., \& Sandberg, E. H. (1994). The coding of spatial location in young children. Cognitive Psychology, 27, 115-148.

Kosslyn, S. M., Koenig, O., Barrett, A., Cave, C. B., Tang, J., \& GABRIELI, D. E. (1989). Evidence for two types of spatial representations: Hemispheric specialization for categorical and coordinate relations. Journal of Experimental Psychology: Human Perception \& Performance, 15, 723-735.

LANDAU, B. (2003). Axes and direction in spatial language and spatial cognition. In E. van der Zee \& J. Slack (Eds.), Representing direction in language and space (pp. 18-38). Oxford: Oxford University Press.

LANDAU, B., \& JACKENDOFF, R. (1993). "What" and "where" in spatial language and spatial cognition. Behavioral \& Brain Sciences, 16, 217-265.

Mandler, J. M. (1996). Preverbal representation and language. In P. Bloom, M. A. Peterson, L. Nadel, \& M. F. Garrett (Eds.), Language and space (pp. 109-169). Cambridge, MA: MIT Press.

Mash, C., Quinn, P. C., Dobson, V., \& Narter, D. B. (1998). Global influences on the development of spatial and object perceptual categorization abilities: Evidence from preterm infants. Developmental Science, 1, 85-102.

McCloskey, M., \& Rapp, B. (2000). A visually based developmental reading deficit. Journal of Memory \& Language, 43, 157-181.

McDonough, L., ChOI, S., \& MandLer, J. M. (2003). Understanding spatial relations: Flexible infants, lexical adults. Cognitive Psychology, 46, 229-259.

NEWCOMBE, N., \& HutTEnLOCHER, J. (2000). Making space: The development of spatial representation and reasoning. Cambridge, MA: MIT Press.

Olson, R. K., \& Attneave, F. (1970). What variables produce similarity grouping? American Journal of Psychology, 83, 1-21.

Piaget, J., \& InHelder, B. (1967). The child's conception of space (F. J. Langdon \& J. L. Lunzer, Trans.). New York: Norton. (Original work published 1948)

QuINN, P. C. (1994). The categorization of above and below spatial relations by young infants. Child Development, 65, 58-69.

QuINN, P. C. (1998). Object and spatial categorization in young infants: "What" and "where" in early visual perception. In A. M. Slater (Ed.), Perceptual development: Visual, auditory, and speech perception in infancy (pp. 131-165). Hove, U.K.: Psychology Press (Taylor \& Francis).

QuinN, P. C. (2002a). Category representation in infants. Current Directions in Psychological Science, 11, 66-70.
QuinN, P. C. (2002b). Early categorization: A new synthesis. In U. Goswami (Ed.), Blackwell handbook of childhood cognitive development (pp. 84-101). Oxford: Blackwell.

QuinN, P. C. (2003). Concepts are not just for objects: Categorization of spatial relation information by infants. In D. H. Rakison \& L. M. Oakes (Eds.), Early category and concept development: Making sense of the blooming, buzzing confusion (pp. 50-76). Oxford: Oxford University Press.

QuINN, P. C. (in press). Developmental constraints on the representation of spatial relation information: Evidence from preverbal infants. In L. A. Carlson \& E. van der Zee (Eds.), Functional features in language and space: Insights from perception, categorization and development. New York: Oxford University Press.

Quinn, P. C., Adams, A., Kennedy, E., Shettler, L., \& Wasnik, A. (2003). Development of an abstract category representation for the spatial relation between in 6- to 10-month-old infants. Developmental Psychology, 39, 151-163.

Quinn, P. C., BhatT, R. S., Brush, D., Grimes, A., \& Sharpnack, H. (2002). Development of form similarity as a Gestalt grouping principle in infancy. Psychological Science, 13, 320-328.

Quinn, P. C., Brown, C. R., \& STREPPA, M. L. (1997). Perceptual organization of complex visual configurations by young infants. Infant Behavior \& Development, 20, 35-46.

QUINN, P. C., BURKe, S., \& RUSH, A. (1993). Part-whole perception in early infancy: Evidence for perceptual grouping produced by lightness similarity. Infant Behavior \& Development, 16, 19-42.

Quinn, P. C., Cummins, M., Kase, J., Martin, E., \& Weissman, S. (1996). Development of categorical representations for above and below spatial relations in 3- to 7-month-old infants. Developmental Psychology, 32, 642-650.

Quinn, P. C., Norris, C. M., Pasko, R. N., Schmader, T. M., \& Mash, C. (1999). Formation of a categorical representation for the spatial relation between by 6- to 7-month-old infants. Visual Cognition, 6, 569585.

Quinn, P. C., Polly, J. L., Furer, M. J., Dobson, V., \& Narter, D. B. (2002). Young infants' performance in the object-variation version of the above-below categorization task: A result of perceptual distraction or conceptual limitation? Infancy, 3, 323-347.

QuinN, P. C., \& Schyns, P. G. (2003). What goes up may come down: Perceptual process and knowledge access in the organization of complex visual patterns by young infants. Cognitive Science, 27, 923935.

REgIER, T., \& CARLSON, L. (2001). Grounding spatial language in perception: An empirical and computational investigation. Journal of Experimental Psychology: General, 130, 273-298.

RIESER, J. J. (1979). Spatial orientation of six-month-old infants. Child Development, 50, 1078-1087.

Rosch, E. H. (1975). Cognitive reference points. Cognitive Psychology, 7,532-547.

SHOLL, M. J., \& Nolin, T. L. (1997). Orientation specificity in representations of place. Journal of Experimental Psychology: Learning, Memory, \& Cognition, 13, 615-628.

SpElKe, E. S., \& Hespos, S. J. (2002). Conceptual development in infancy: The case of containment. In N. L. Stein, P. J. Bauer, \& M. Rabinowitz (Eds.), Representation, memory, and development: Essays in honor of Jean Mandler (pp. 223-256). Mahwah, NJ: Erlbaum.

TALmy, L. (1983). How language structures space. In H. L. Pick, Jr. \& L. P. Acredolo (Eds.), Spatial orientation: Theory, research, and application (pp. 181-238). New York: Plenum.

Xu, F., \& CAREY, S. (1996). Infants' metaphysics: The case of numerical identity. Cognitive Psychology, 30, 111-153.

(Manuscript received July 8, 2003; revision accepted for publication January 6, 2004.) 